\title{
Leveraging pharmaceutical preparedness for epidemic outbreaks in the West African sub-region: a review
}

\begin{abstract}
Background: The increasing waves of political instability around the world today lead to increasing number of internally displaced persons and refugees within and across local and international borders. In line with international health and global best practices, short, medium, and strategic plans towards combating epidemic outbreak become imperative.
\end{abstract}

Objective: The study explored and discussed factors that improve Pharmaceutical Preparedness for Epidemic Outbreaks in the West African sub-region.

Methods: A narrative overview of literature was conducted to articulate and synthesize necessary findings obtained from review of relevant literatures. Literature search was carried out in International Pharmaceutical Abstracts, Medline, authoritative texts, and Google Scholar using keywords individually and in strings to explore the pharmaceutical for epidemics and leveraging factors. Only officially, published documents relevant to the subject and articles with standard methods and procedures were considered eligible for the study. All the documents used were published in English language. Study lasted between October and December 2016.

Results: Study showed that $60 \%$ of all pharmaceutical production in the West African sub-region takes place Nigeria and her population exceed $50 \%$ of the entire human population in the sub-region. It identified human resources development and utilization, research and development, and improved capacity utilization as the driving forces that can boost pharmaceutical preparedness to epidemic outbreak. Other factors include strategic planning for sourcing and production of scarce drugs and vaccines, efficient drug supply system, provision of enabling business climate through government policy, infrastructural development, strict regulatory framework, and political stability.

Conclusion: Ensuring pharmaceutical preparedness in the sub-region is invaluable considering the fact that epidemics move in a cycle and come unannounced. The factors that improve epidemic prepared include improved manpower development that will promote technology transfer, and capacity utilization. Provision of enabling sociopolitical, and economically stable and viable environment with sound policies will reposition the pharmaceutical sector in the sub-region for epidemic outbreak and other eventualities.

Keywords: epidemic preparedness, pharmaceutical sector, west Africa, disease outbreak, drug availability
Volume 6 Issue I - 2017

\author{
Ogbonna Brian O \\ Department of Clinical Pharmacy and Pharmacy Management, \\ Faculty of Pharmaceutical Sciences, Nnamdi Azikiwe University, \\ Nigeria
}

\author{
Correspondence: Ogbonna Brian O, Department of \\ Clinical Pharmacy and Pharmacy Management, Faculty of \\ Pharmaceutical Sciences, Nnamdi Azikiwe University, Nigeria, Tel \\ +2348037794206, Email bo.ogbonna@unizik.edu.ng
}

Received: April 0I, 2017 | Published: June 13, 2017
Abbreviations: GDP, gross domestic product; ECOWAS, economic community of west african states; CET, commission external tariff; WHO, world health organization; GMP, good manufacturing practice; FDA, food and drug agency; IDP, internally displaced persons

\section{Introduction}

\section{Background}

Africa has $75 \%$ of global HIV/AIDS burden and malaria $90 \%$ of approximately 300-500 million cases of malaria in the world annually take place in the region, an indication for huge demand for drugs, vaccines and essential pharmaceuticals. High incidence of communicable disease burden and Neglected Tropical Diseases still abound in Africa. African countries have 19 out of the 20 countries of the world with the highest maternal mortality ratio and record the highest neonatal death rate globally. This scenario coupled with poor health indices underscores the ever-increasing need for drugs and pharmaceuticals. Indigenous pharmaceutical manufacturers in Africa produce approximately $25 \%$ to $30 \%$ of their drugs and $10 \%$ of their medical consumables. The 2016 World Health Organization's report showed that majority of West African countries has life expectancy below 65.0years. Nigeria and Sierra Leone scored 54.5 and 47.5 respectively. The mortality, health system indicators and diseases statistics from 194 countries shows that this region is plagued by illness and death from key diseases, and poor financial investment on health. This was complicated by abysmal poor budgetary allocation for health and weak health systems. Financial and material resources have been mobilized globally since 2008 to improve emergency preparedness to epidemics. ${ }^{1,2}$ Statistics show that $60 \%$ of all pharmaceutical production in the West African sub-region takes place in Nigeria. However, out 
of approximately 120 indigenous drug manufacturers in Nigeria, capacity utilization was estimated to be $40 \%{ }^{3}$ This study explored and discussed ways of improving Pharmaceutical Preparedness for Epidemic Outbreaks in the West African sub-region.

\section{Methods}

A review of the state of pharmaceutical manufacturing sector in West Africa was undertaken. Literature search was conducted using the keywords as search terms and their combinations, truncations, rephrasing, adjacent searching, and strings of terms. Literature search was conducted in International Pharmaceutical Abstracts, Medline, authoritative texts, and Google Scholar. Literature search was additionally conducted in institutional and private websites for official and published documents and relevant literatures relevant to the topic. Only officially, published documents relevant to the subject and articles with standard methods and procedures were considered eligible for the study. All articles used were published in English and where more than one literature show similar information preference was given to the most recent one. Officially, published documents from reorganized websites were used. The number of records identified through database search was 58 while 18 additional records were identified through authoritative texts and hand search, which gave rise to 76 records. The 76 articles and materials were screened to remove 13 duplicates to give rise to 63 articles assessed for eligibility. Based on eligibility criteria, 51 full text articles were excluded due to inconclusive findings, reporting bias, non-standardized methods, inconclusive and poorly defined study selection. This gave rise to 25 materials used for the study.

\section{Result and discussion}

The United Nations High Commission for Refugees has trained public health officers from around the world on the development and establishment of outbreak preparedness to epidemics and capacity building for communicable diseases control. Disease outbreak is very common in slums and refugee camps, which make them focal points for epidemics that ultimately invade the wide population in the resident countries and region. These measures are very significant since poorly handled epidemics of highly infectious organisms can assume pandemic proportion and become more devastating. ${ }^{4} \mathrm{~A}$ strategic aspect of epidemic preparedness and response in public health borders on effective synergy with all key players. This will be tailored to national and international outbreak and response at all levels while ensuring prompt and improved cost-effective preparedness and response activities. ${ }^{4,5}$

It is therefore necessary to herald a wake-up call for pharmaceutical preparedness to epidemics in the sub-region. Machineries must be in place to contain epidemics before the arrival of foreign interventions and aids due to rapid spread of epidemics to minimize casualties while containing the menace. Statistics showed that most countries in the West African sub-region have poor health percentage of gross domestic product (GDP) occasioned by life expectancy ranging from 47.5 to $62.5 y e a r s$ against their counterpart in the civilized world who have life expectancies of approximately 80 years and high health percentage of GDP as shown in Table 1 below. This is attributable to myriads of factors ranging from poor socioeconomic status to political instability, poor human resources development, infrastructural neglect, weak policies, and institutional structures. This predisposes the sub-region to capacity underutilization and poor economic status that leaves her at the mercies of foreign aids to break even. Consequently, casualty figures are usually alarming during disaster outbreaks and epidemics in waiting for external aids and interventions in form of vaccines, drugs, consumables, and manpower from outside the region. ${ }^{5,6}$

Table I A table comparing some health indicators of West African countries to two developed countries

\begin{tabular}{|c|c|c|c|c|c|c|c|c|c|c|c|}
\hline SN & Indicators & Senegal & $\begin{array}{l}\text { Sierra } \\
\text { Leone }\end{array}$ & Nigeria & Ghana & Gambia & Liberia & Togo & Benin & UK & USA \\
\hline I & $\begin{array}{l}2015 \text { Population } \\
\text { (million) }\end{array}$ & 15.1 & 6 & 182 & 27.5 & 2 & 4.5 & 7.3 & 10.8 & 65 & 222 \\
\hline 2 & $\begin{array}{l}2015 \text { Life } \\
\text { expectancy (Years) }\end{array}$ & 67 & 47.5 & 54.5 & 62.5 & 61 & 61.5 & 60 & 60 & 81 & 79.5 \\
\hline 3 & $\begin{array}{l}\text { Health \% of GDP } \\
\text { in } 2014\end{array}$ & 4.7 & II.I & 3.7 & 3.6 & 7.3 & 10 & 5.2 & 4.6 & 9.1 & I7.I \\
\hline
\end{tabular}

Evidence-based research, health services, and equity in the sub-region

Pharmaceutical preparedness towards epidemic outbreak entails tailoring health services and facility planning to sustained, efficient, and effective drug supply management. Gross inequity in health between developed and developing countries, within regions and countries necessitated the concept of equity in health in the Alma Ata Declaration of $1978 .^{7}$ Equity in public health services is promoted by political commitment, monitoring of equity, information and research, policy formulation, community involvement and inter-sectoral action. Health research broadly divided into two could be country specific or of global perspective. Country specific health research borders on situation analysis, which defines health and disease distribution and identify determinants and risk factors through statistics, epidemiology and sociology. It could be health policy and system inclined and explores ways of enhancing efficiency and effectiveness of health systems and interventions, promotion of cost-effectiveness approach, and provision of framework for evaluation of equity and health policies through economics, management sciences, political sciences, communication, and social sciences. Global health research focuses on developmental and basic research to develop new technologies for disease prevention and management, advance knowledge of basic biology with potential application in tackling human diseases, and enhance understanding of human behaviors, poverty, individual, or group dynamics. ${ }^{89}$ Health research provides useful information on disease trends and patterns used in forecasting, short, medium, and strategic planning for epidemics outbreak. National drug policies provide effective framework for managing drug supplies for cost-effective availability and affordability of essential drugs..$^{8-10}$ Research and development is a driving force in any viable and dynamic economy. Research and development should target product development and standardization to attain global best practice and standards. It will make her products competitive to target key and 
viable markets in developed economies and build her asset base for diversification to match any form of challenge and pressure during disaster and epidemic outbreaks. It promotes the development of new and innovative products, which promote healthy competition in the sector. ${ }^{9}$

\section{Pharmaceutical sector manpower need in the sub- region}

Epidemics predominantly occur in underdeveloped countries with poor health indices and weak capacity for health services. Drug companies have minimal incentives to develop drugs and vaccines for poor underdeveloped countries who cannot afford the patent. International donors prefer investing in drugs for diseases with potentials of assuming epidemic or pandemic dimension, which could be devastating to already weak health systems. A study carried out in West African sub-region indicated that the Gambia, Ghana, Liberia, Nigeria, and Sierra Leone have gross human resources need for provision of pharmaceutical services in the sub-region, which is fundamental to production, availability, and prudent management of drug supply services. Development of human resources for the pharmaceutical sector will provide strategic focus for access to medicines, efficient health care delivery, and good financing. This underscores the need for health system strengthening, effective and prudent use of resources especially in the sub-region already plagued with poor health indices. It will ensure availability and affordability of essential drugs, vaccines and other medical consumables to meet the ever-increasing demand while maintaining surplus in reserve for epidemic outbreak. ${ }^{10,11}$

\section{Approach to scarce drugs, consumables, and vaccines for rare diseases and epidemics}

Epidemics sometimes move in a cycle reoccurring from one time and season to another and disease outbreak in a remote locality today may assume an epidemic or pandemic proportion threatening an entire region and beyond. This was obvious in the Ebola epidemic that occurred in the West African sub-region. The outbreak stated in 1976 but without much political and diplomatic will to combat the scourge until nearly four decades in 2014 when the outbreak became a threat not only to the sub-region but to other distant regions and beyond. WHO should have a strategic plan for drugs and vaccine development against seemingly unpopular diseases today, which can become devastating tomorrow? This is especially when the world has become a global village with weak international health services where disease outbreak can easily spread. ${ }^{12}$ Framework and funding for drug and vaccine development should be encouraged irrespective of the regions involved and their economic or developmental status. Pooling resources from member nations like the principle of health insurance for servicing such capital-intensive projects in collaboration with multinationals can eliminate the problems faced by poor and individual nations in handling such capital projects. ${ }^{13,14}$ Nations can team up at sub-regional levels. A framework should be in place in drug and vaccines development for neglected tropical diseases.

\section{Raw material development and review of economic policies}

Raw material development has been neglected in our pharmaceutical sector today. Pharmaceutical industries can enter into partnership with research institutions and petrochemical industries for development of raw materials. This could be carried out in phases for development of simple to more complex raw materials through a strategic plan. Due to trade liberalization with minimal trade and export inhibition to exportation globally, major manufacturers who largely determine the market forces and trends determine raw material prices. This was facilitated by the recent adoption of the Economic Community of West African States (ECOWAS) Commission External Tariff (CET). Pharmaceutical manufacturers in the sub-region should plan strategically to meet up with global standards for obtaining World Health Organization (WHO)'s Good Manufacturing Practice (GMP) compliant status to enable them compete favorably with their counterparts from Europe, Asia and America. This should be in addition to meeting the national and sub-regional demands for pharmaceuticals. ${ }^{15,16}$ Pharmaceutical companies should upgrade their facilities world standard benchmarks like the European Regulations and Food and Drug Agency (FDA) specifications while focusing production to explore over the counters, core ethical's, and other important health care consumer products. They should go beyond meeting local consumption demands to cashing in on export share.

Partnership with research institutions and organizations can bridge this gap. Most indigenous pharmaceutical manufacturers in the west African sub-region below 1 Billion Dollars and contributing almost nothing to Gross Domestic Product (GDP) against their counterparts in Europe, Asia and United States. Without strategic plan for pharmaceutical raw material development, the pharmaceutical manufacturers in the sub-region will be at the mercies of numerous mono product plants that leverage on volume sales and non-inhibition to export to push pharmaceutical raw materials around the world. These capital projects need to be tackled from a short, medium to strategic framework. The federal governments should review their economic policies and foreign exchange practices to promote the activities of indigenous pharmaceutical manufacturers. Tax incentives, low interest loans, and elimination of multiple taxations will be invaluable to the industries. Most pharmaceutical industries in the West African sub-region are plagued by multiple taxations, and over inflation of taxes. ${ }^{12,17}$ These, coupled with already hash economic climate, which makes the sub-region very economically hostile for operations. This will promote manufacturing and export which catalyzes industrial and economic growth.

\section{Repositioning regulatory agencies and tackling unfair regulatory practices}

Regulation of massive importation of finished products especially those manufactured locally to promote local content development. Dumping of substandard, unneeded, and other products flood the local markets with undesired products that adversely affect price control regulatory requirements should be clearly defined and unambiguous. The regulatory system should be sanitized, well equipped, and funded like their counterparts in Europe, and United States. A good example is the FDA. Routine manpower training and collaboration should be encouraged through diplomatic ties and understanding. Donation of drugs and vaccines should be based on clearly defined based on demands and the prevailing needs nationally and within the sub-region. Government to encourage endogenous pharmaceutical manufacturers in our sub-region should use moratorium. This could be varied from short to medium term based on the developmental milestones of the companies and their capacity. ${ }^{18,19}$

\section{Effective and efficient drug supply management and system strengthening}

An efficient drug production system in addition to effective capacity utilization in production should have and effective and 
efficient logistic and supply chain system for efficient supply on demand. To promote efficient pharmaceutical preparedness within the sub-region, government policies must favor the critical aspects of drug selection, procurement, distribution, use, financing and sustainability, information management, organization, and human resources management. ${ }^{7,8}$ These factors are necessary for efficient and effective supplies during epidemics and disaster response because drugs unlike other commodities are expensive, save lives, improves health, encourage trust and participation in health care delivery and serves as the final link between caregivers and facilities. Huge gap exist between the need for essential drugs and access. This is compounded in epidemic outbreak where there is high demand within very limited time in life threatening conditions..$^{20,21}$ Effective tracking and control of these factors through enabling government policies and prompt implementation of same will promote pharmaceutical preparedness in the sub-region.

\section{Political stability and sustained infrastructural development}

Most of the West African countries have had their shares of civil war, corruption, and political instability while others have lingered until date. Nigeria and Liberia have histories of corruption and civil war. Sierra Leone and Guinea have histories of long lasting corruption, which is taking its toll on the entire system. ${ }^{22,23}$ The operation of terrorist group called Boko Haram in north east Nigeria has further left her with humanitarian condition of internally displaced persons (IDP) in different resettlement and IDP camps. The Gambia is presently grappling with a state of political instability following their last presidential election. Some of the countries in the West African sub-region have a history of poor infrastructural development associated with longyears of military rule and systemic corruption. Electricity, good road network, portable water supply, and adequate security, which are driving forces of the economy, are nonexistent in most West African countries. These have negatively affected investors as limitations to potential investors.

\section{Limitations}

Some relevant materials and articles may have been incompletely retrieved or discussed. Bias may not have been completely ruled out at the study or outcome level.

\section{Conclusion}

Repositioning the pharmaceutical sector in the West African subregion will promote access to medicines for the generality of the public and reposition her for preparedness during emergencies and epidemic outbreaks while promoting health care delivery within the sub-region and beyond. There is need to step up capacity building and utilization in the pharmaceutical sector. Massive investment into research and development that will aid raw material development and diversification of product lines while improving on quality and standards to meet international best standards of practice and quality. Embracing the fundamental principles of logistics and drug supply management at all strata of the pharmaceutical value chain from the manufacturers to the consumers through institutional strengthening, management information systems, monitoring and evaluation will promote availability of the products even to very remote places in times of emergency. Repositioning the regulatory agencies will not only safeguard the sector but also prevent the uncontrolled dumping of unwanted and substandard products that negatively affect the market dynamics. Government should review their policies and expedite actions at implementing the policies that will promote the sector. This should be without prejudice to creating an enabling and conducive political, infrastructural, and socioeconomic environment for effective business operations to serve as driving force for the pharmaceutical sector.

\section{Acknowledgements}

No funding was provided for this study. However, I am indebted to the staff and management of the Department of Clinical Pharmacy and Pharmacy management, the Department of Community Medicine and the University community of Nnamdi Azikiwe University for provision of a conductive work and learning environment throughout the period of this study.

\section{Conflict of interest}

Author declares that there is no conflict of interest.

\section{References}

1. The African Regional Health Report, World Health Organization (WHO). The Health of the People. 2016;96:1-2.

2. Global health observatory. World Health Organization (WHO); 2016.

3. Pharmaceutical Sector Profile Nigeria Global UNIDO Project: Strengthening the local production of essential generic drugs in least developed and developing countries. United Nations Industrial Development Organization (UNIDO). Vienna; 2011:1-64.

4. Epidemic Preparedness and Response in Refugee Camp Settings Guidance for Public Health Officers. The United Nations High Commission for Refugees (UNCHR), Geneva, Switzerland; 2011:1-15.

5. Ayodele A 'Ebola stops with me', Freetown, Sierra Leone; Simon Davis/ DFID.

6. Emerging Pandemic Threats program. 2016.

7. Managing Drug Supply. 2nd ed. Revised and expanded. In collaboration with WHO. Part 1 \& 4. Management support systems, Management Science for Health. 1997. p. 1-496.

8. Foster SD. Supply and use of essential drugs in sub-Saharan Africa: some issues and possible solutions. Soc Sci Med. 1991;32(11):1201-1218.

9. Adetokumbo OL, Herbert MG. Short Textbook of Public Health for the Tropics. 4th ed. Organization of Health Services. 2004;10:283-308.

10. F Tayo. Human resources needs for the provision of pharmaceutical services in the West African sub-region. West African Journal of Pharmacy. 2007;20(1):4-7.

11. Lannes L. Revolutionalizing Africa's Pharmaceutical Industry. African Development Bank Group; 2014:1

12. Overview on Ebola Research Update February 2015. European Commission Directorate-General for Research and Innovation Directorate E - Health E.3 - Fighting Infectious Diseases and Global Epidemics. 2015:1-46.

13. How to get vaccines and drugs. 2016.

14. Ebola Response, Recovery And Resilience In West Africa Call For Partnership Concept Papers Under Existing Fy 2014/ Fy 2015 Global Development Alliance (Gda), Annual Program Statement (Aps) No: Aps-Oaa-14-000001, U.S. Agency For International Development Announcement, USA; 2015;1-33.

15. Indicators For Monitoring National Drug Policies. World Health Organization/Action Program on Essiential Drugs (WHO/DAP). Geneva; 1994. 
16. Banjoko S. Nigerian manufacturing sector: Bumpy past and shaky future: what options for survival. The text of an inaugural lecture at the Department of Business Administration. University of Lagos; 2009.

17. Okelola K. Pharmaceutical Manufacturers Group of the Manufacturers Association of Nigeria: An Overview of the Pharmaceutical Sector. PMGMAN, Abuja; 2009.

18. Report of a nationwide assessment of installed and utilized capacity of the local drug manufacturing companies. Federal Ministry of Health, Abuja/The Presidential Committee on the Pharmaceutical Sector Reform; 2008.

19. National Agency for Food, Drug Administration, and Control, Manufacturers Association of Nigeria. The Evolution of Drug Production (MAN/NAFDAC) in Nigeria; 2011:1-2.

20. EU Response to Ebola epidemic in West Africa, European Commission. Humanitarian Aid and Civil Protection, ECHO Factsheet - Ebola in West Africa; 2016:1-3.
21. Roache S, Gostin SL, Hougendobler D, et al. Lessons from the West African Ebola Epidemic: Towards a Legacy of Strong Health Systems. O’Neill Institute for National and Global Health Law; 2016. p. 10-13.

22. Emergency Request Justification. Department of State, Foreign Operations, and Related Programs. Department of State United States of America; 2015. p. 1-26.

23. Public Health Emergency Medical Counter measures Enterprise (PHEMCE). Strategy and Implementation Plan, U.S. Department of Health and Human Services, USA; 2014. p. 1-56.

24. Ebola Response, Recovery And Resilience In West Africa Call For Partnership Concept Papers Under Existing Fy 2014/ Fy 2015 Global Development Alliance (Gda), Annual Program Statement (Aps) No: Aps-Oaa-14-000001, U.S. Agency For International Development Announcement, USA; 2015. p. 1-33.

25. United Nations Office for the Coordination of Humanitarian Affairs. Nigeria: northeast crisis situation report no. 1; 2015. 